\title{
What Can We Learn From Dynamics Of Nearby Galaxies To Study Distant Galaxies?
}

\author{
B. Epinat ${ }^{1}$, P. Amram ${ }^{1}$ and C. Balkowski ${ }^{2}$ \\ ${ }^{1}$ Laboratoire d'Astrophysique de Marseille, Observatoire Astronomique Marseille-Provence, \\ 2 place Le Verrier, 13248 Marseille Cedex 04, France - email: benoit.epinat@oamp.fr \\ ${ }^{2}$ Laboratoire Galaxies Etoiles Physique et Instrumentation, Observatoire de Paris-Meudon, \\ 5 place Jules Janssen, 92195 Meudon Cedex, France
}

This is an ongoing project.

During last few years, new instruments like GIRAFFE (Flores, H., Hammer, F., Puech, M., et al., 2006, A\& $\&$ 455,107) or SINFONI (Förster Schreiber, N.M., Genzel, R., Lehnert, M.D., et al., 2006, ApJ 645,1062) have began investigations on 3D kinematics of high redshift galaxies. This is just the head of the iceberg as Extremely Large Telescope will allow this kind of study on a larger sample. By now, it is important to be able to recover the actual kinematic parameters, and overall to disentangle evolutionnary effects from distance effects.

In this aim, we use the kinematical reference sample of 200 nearby galaxies GHASP (Garrido, O., Marcelin, M., Amram, P., et al., 2005, MNRAS 362,127, and reference therein) for which we have seeing-limited 3D data cubes obtained with a Fabry-Perot scanning $\mathrm{H} \alpha$ line. GHASP is a complete sample that covers a large range of luminosities and morphological types, except ellipticals.
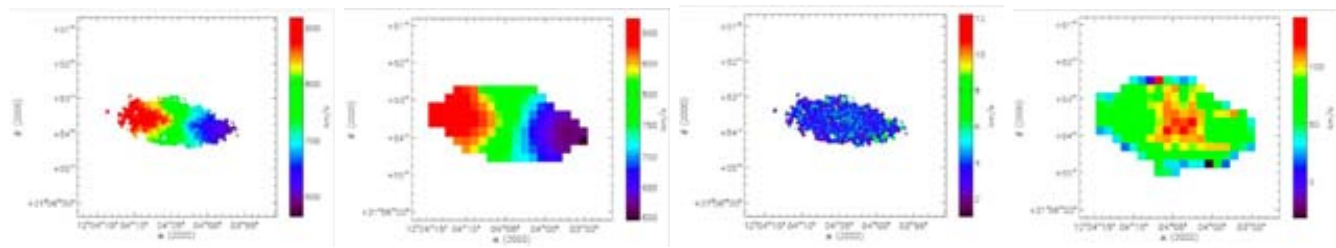

Figure 1. Velocity field (left) and velocity dispersion field (right) of UGC 7045 at $\mathrm{z}=0$ ( $1^{\text {st }}$ and $3^{\text {rd }}$ from the left) and $\mathrm{z}=0.4\left(2^{\text {nd }}\right.$ and $4^{\text {th }}$ from the left $)$.

In figure 1, UGC 7045 has been projected from redshift 0.0026 to redshift 0.4 , using a seeing of 0.3 " (assuming adaptadive optics)and with a pixel of 0.14 ". No cosmological effects have been taken into account and no noise has been added. Velocity fields and velocity dispersion fields are displayed. From velocity fields, we can recover parameters such as position angle, inclination, rotationnal velocities, center position but also inner slope of the rotation curve. As resolution is lowered, accuracy on parameters determination is worse. We show that we need a ratio between the seeing and the size of the galaxy at least of 20 to trace such a rotation curve poorly biased. It is also clear that velocity dispersion maps in the central regions increases when resolution decreases.

This work shows how adaptative optics will be usefull to characterise velocity fields at high redshifts, but also the cares that have to be taken when concluding on a classification based on velocity fields or velocity dispersion features. It will be completed by the study of the whole GHASP sample using kinemetric methods as presented in Krajnović, D., Cappellari, M., Tim de Zeeuw, P., et al., 2006, MNRAS 366,787. 